\title{
The Shanzhai City
}

\author{
Teis De Greve \\ Social Spaces \\ LUCA School of Arts \\ Genk, BE \\ teis.degreve@luca-arts.be
}

\author{
Bieke Zaman \\ Mintlab/IMS \\ KU Leuven \\ Leuven, BE \\ bieke.zaman@kuleuven.be
}

\author{
Jessica Schoffelen \\ Social Sciences, KU Leuven \\ Inclusive Society, UC Leuven- \\ Limburg \\ Leuven, BE \\ jessica.schoffelen@ucll.be
}

\begin{abstract}
Smart cities are often criticised as top-down and technocratic. However, initiatives that are more citizen-centric have difficulties contending with the prevalent technology-driven discourse. From a design perspective, this points towards a delicate balance between an approach that is critical enough to resist assimilation yet constructive enough to have a sustainable impact. This paper aims to explore and articulate this balance through the discussion of shanzhai, a Chinese phenomenon on the fringe of the global market economy. More particularly, we highlight three qualities of shanzhai by linking these to the case study of a smart bicycle lock, followed by a discussion in terms of their agonistic qualities. These three qualities of shanzhai, the marginalised people it caters, its hybrid aesthetic and its position in the market, could inform a design practice that critically yet constructively engages with technology in the context of smart cities.
\end{abstract}

\section{KEYWORDS}

Smart cities, smart citizens, shanzhai, adversarial design

\section{ACM Reference format:}

Teis De Greve, Bieke Zaman and Jessica Schoffelen. 2019. The Shanzhai City. In 31ST AUSTRALIAN CONFERENCE ON HUMAN-COMPUTERINTERACTION (OZCHI'19), December 2-5, 2019, Fremantle, WA, Australia. ACM, New York, NY, USA, 5 pages.

https://doi.org/10.1145/3369457.3369529

\section{INTRODUCTION}

The smart city is often criticised as top-down and technocratic, serving the interests of corporations and governments rather than those of the people who inhabit the city $[10,15,32]$. In response, designers and academics, sometimes backed by genuine interest from government and businesses, have explored approaches that facilitate a more citizen-centric conception of the smart city. These endeavours have taken different shapes, from more mainstream

Permission to make digital or hard copies of part or all of this work for personal or classroom use is granted without fee provided that copies are not made or distributed for profit or commercial advantage and that copies bear this notice and the full citation on the first page. Copyrights for third-party components of this work must be honored. For all other uses, contact the Owner/Author.

OZCHI'19, December 2-5, 2019, Fremantle, WA, Australia

(C) 2019 Copyright is held by the owner/author(s).

ACM ISBN 978-1-4503-7696-9/19/12.

https://doi.org/10.1145/3369457.3369529 to more critical design practices. However, it appears difficult to contend with the prevalent, technology-driven discourse.

On the one hand, initiatives like city apps [e.g. 24,25], hackathons [e.g. 1] or citizen sensing projects [e.g. 9] are attempts to involve citizens in smart city developments, aiming to shift the discourse from smart cities to smart citizens [13]. While such initiatives certainly add to a more citizen-centric conception of the smart city, Cardullo and Kitchin [4] have questioned whether this can bring forth a true shift in the prevailing technocratic discourse. From their analysis of a number of citizen-centric initiatives, the authors note how "smart citizens perform within the bounds of expected and acceptable behaviour, rather than transgressing or resisting social and political norms" [4]. They conclude their analysis with a call to re-politicize the smart city.

On the other hand, more political design practices engaging with technological futures, like critical or speculative design (CSD), have also come under scrutiny $[3,26,33]$. The visions that are depicted in critical or speculative design projects are often dark and dystopian. If such projects fail to successfully communicate their critical position, they are reduced to yet another dystopian science fiction, likely to estrange the larger audience [26].

From a design perspective, this tension illustrates a delicate balance between citizen-centric approaches to the smart city that on the one hand are critical enough to resist assimilation and on the other hand are constructive enough to have a sustainable impact.

This paper aims to explore and articulate this balance through the discussion of shanzhai, a Chinese phenomenon on the fringe of the global market economy. First, we will frame the notion of shanzhai, and elaborate why it could be useful from a design perspective. This is followed by a brief section on agonism and adversarial design [7] to provide a theoretical foundation for the discussion of this paper. We then present the case study of a prototype for a smart bicycle lock, where the method of critical making [28] will be put forward as a way to explore the qualities of shanzhai through material production. This will lead to a discussion, which will highlight three qualities of shanzhai that could inform a design practice that critically yet constructively engages with technology in the context of smart cities. These three qualities will each be discussed by relating them to both the case study of the smart bicycle lock, and to the theory on adversarial 
design. Finally, a closing section will look forward to future directions for this project.

\section{SHANZHAI \& AGONISM}

The term shanzhai is often used in a derogatory way, referring to Chinese counterfeit products of inferior quality-specifically consumer electronics, produced in small factories in the rural area around the city of Shenzhen.1 While it is true that many shanzhai manufacturers started out by copying existing devices, some have evolved to produce a number of highly original products [21]. The best way to grasp the phenomenon of shanzhai, might be through these products. The most telling examples can be found in the mobile phone sector, as documented in the project "Shanzhai Archaeology" by the DISNOVATION.ORG working group. One of the examples is a phone tailored for the Chinese elderly who like to dance together on public squares. It features a large speaker to play loud music outdoors, a small support stand to put it in front of the dancers, a flashlight for walking home on dimly lit roads and it comes preloaded with several gigabytes of old-fashioned songs. Other examples include: a Prisoners Phone with voicechanger that is $99 \%$ plastic and difficult to detect during security checks, a Power Bank Phone to face power cuts in Ghana, or a Buddha Phone that features a virtual altar $[6,23]$.

Academic work on shanzhai has mostly been concerned with the aspect of economic transition [e.g. 5,21,34], and the analysis of shanzhai as an innovation ecosystem that is familiar, yet different from Western open-source and maker culture [e.g. 19,22]. With the exception of the work of DISNOVATION.ORG [6,23], shanzhai has largely been underexplored from a design perspective. Nevertheless, shanzhai has a distinct characteristic; as "neither straightforward counterculture nor pro-system" [22], it seems to uphold a delicate balance between an approach to design that is critical enough to resist assimilation yet constructive enough to have a sustainable impact, which is what this paper set out to explore and articulate. To give shape to this discussion, we will relate shanzhai to Carl DiSalvo's work on adversarial design.

\section{Adversarial design}

In his work on adversarial design, DiSalvo theorises and exemplifies how design can evoke and engage with political issues [7]. Drawing on the work of political theorist Chantal Mouffe [27], adversarial design centres around agonism-a condition of contestation and dissensus-as a fundamental aspect of democracy. Thus, design that engages with political issues, is design that continuously strives to question the status quo and provide opportunities for contestation and productive conflict.

Although shanzhai might not be inherently political-at least it does not explicitly aim to be so-its ambiguous position between counterculture and pro-system does place it in the realm of adversarial design. Before moving on to a discussion on shanzhai in terms of its agonistic qualities, the following section will present a short case study of a smart bicycle lock. As this paper aims to explore the qualities of shanzhai from a design perspective, the inclusion of this case study aims to bring the discussion beyond a mere theoretical level.

\section{PROTOTYPE: A SMART BICYCLE LOCK}

\section{Methodology}

This section of the paper will discuss the design of a prototype for a smart bicycle lock, made by the first author of this paper during a two-week stay in Shenzhen. The process of designing this prototype played an important role in both exploring and defining the qualities of shanzhai. This reciprocity between physical and conceptual exploration, is what Matt Ratto calls critical making, a mode of work "focused on using material production-making things-as part of an explicit practice of concept elaboration within the social study of technology" [28]. Put more simply, prototypes become "things to think with" [12].

The prototype draws on observations of a smart city infrastructure in Shenzhen; the dockless bicycle sharing systems that are ubiquitous in the city. The next section will discuss this context in more detail, followed by a description of the prototype. The discussion will then be continued by drawing on the design process to highlight three qualities of shanzhai and their relationship with the concept of adversarial design.

\section{Context}

Like in other major Chinese cities, dockless bicycle sharing systems rapidly gained popularity in Shenzhen. In August 2017, 890.000 bikes from 10 different companies were in use, accounting for 4.53 million bike trips a day [17]. The number has since dropped due to intense competition between the different providers, along with increased pressure from government regulations [18]. However, when visiting Shenzhen in April 2019, the ubiquity of the sharing bikes still was noticeable. Two adjacent observations could be made: Shenzhen's apparent lack of bicycle infrastructure, and the large number of unlocked, broken, abandoned, vandalised and stolen bikes that could be found around the city. This seemed to point towards an intricate relationship between sharing bike providers and users. The companies behind the systems leech on public space for their profit, without contributing to a suitable cycling infrastructure. Users would respond with disregard towards the sharing system, resulting in an accumulation of damaged and discarded bikes around the city.

One practice that particularly stood out, was that of appropriating sharing bikes, either by making the QR code unreadable or putting a proprietary lock on the bicycle (see Figure 1). Such bikes could notably be found near large construction sites around the edge of the city. From a top-down smart city

\footnotetext{
${ }^{1}$ shan: mountain, zhai: stronghold, historically referring to remote villages ruled by regional warlords, far away from official control
} 
perspective, this practice is undesirable. However, the fact that the appropriated sharing bikes are widespread, points to a potential group of users that do not fit in the bounds of expected an acceptable use of the system. The prototype of the smart lock that will be described in the following paragraphs departs from this situation.
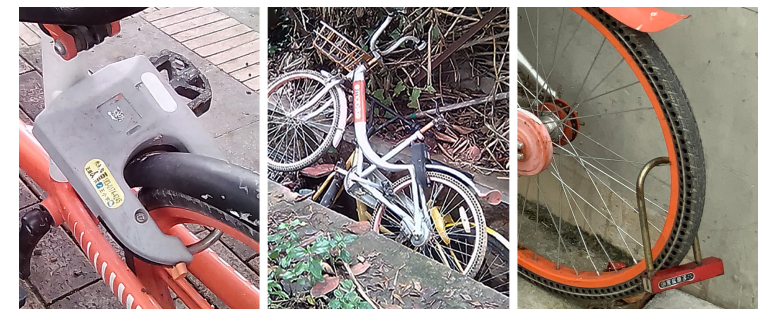

Figure 1: Vandalised, broken and stolen sharing bikes

\section{The prototype}

The prototype of this smart lock is built around a standard U-lock from a sports equipment retail chain. The U-lock is extended with a 3D-printed enclosure, its shape resembling that of a barcode scanner, that sits around the crossbar section of the lock. The enclosure is sanded, polished and spray painted, bringing its appearance closer to a moulded mass-production item rather than a 3D-print, the latter being more commonly associated with early prototypes and maker culture. The enclosure contains electronic components, most notable a QR code scanner, a GPRS-module for internet connection, an Arduino microcontroller and a rechargeable battery (see Figure 2). On the microcontroller, a simple program based on open-source modules takes care of decoding the data from a scanned $\mathrm{QR}$ code and sending and receiving requests to and from the API of the sharing bike provider. ${ }^{2}$
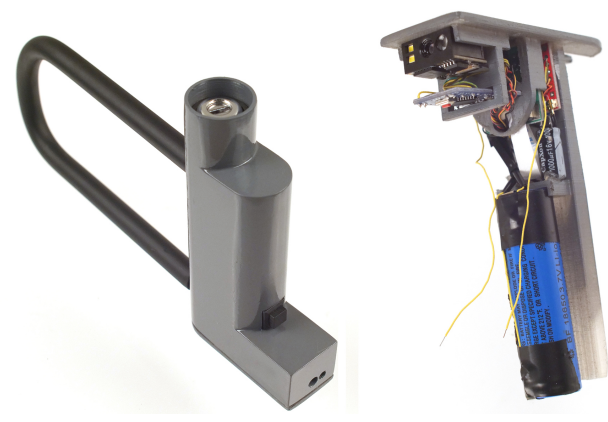

Figure 2: The lock with a close-up of the electronics inside

The result is a hybrid design to support the act of appropriating a sharing bike. Without the need to download a smartphone application or register an account, a person can use the prototype to open the electronic wheel lock of a sharing bike, drive the bike, and park it (see Figure 3). After parking, the integrated U-lock in

${ }^{2}$ Depending on the security protocols used by the sharing bike provider, hacking a smart lock ranges from very easy to extremely hard. This part of the project was not the prototype can be used to lock the bike, ensuring it will not be used or moved by anyone else in the meantime.

The prototype was documented through photographs, both in a studio as during use. Additionally, the smart lock was presented during a monthly meetup event for start-ups, makers and designers in Seeed, a well-known hardware innovation lab in Shenzhen.

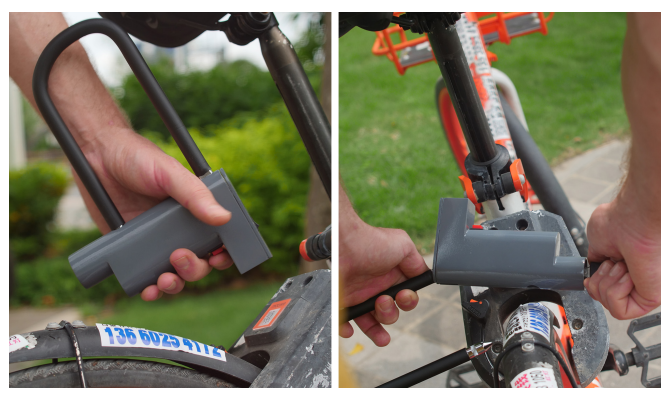

Figure 3: The lock in use

\section{DISCUSSION}

The prototype of the smart lock was valuable to connect with shanzhai on a material level and articulate the qualities that are relevant from a perspective of design. Three qualities that could inform a design practice that critically yet constructively engages with technology in the context of smart cities will be discussed in more detail here. Each will be linked to the case study of the smart bicycle lock followed by a discussion in terms of their agonistic qualities. These are: (1) shanzhai caters people whose demands are not met or ignored by the formal market; (2) shanzhai has a hybrid aesthetic; and (3) shanzhai is a commercial practice.

\section{Marginalised people}

A first quality of shanzhai is that it caters people whose demands are not met or ignored by the formal market. It is "offering solutions to marginal problems" [23].

The smart bicycle lock takes the common practice of appropriating sharing bikes as a starting point. Rather than counteracting or discouraging, the design of the lock highlights this practice. Through its particular functionality and design, the lock tells about the people that would use it-and by extension the circumstances under which its use could emerge. The designed object is a confirmation and reminder of a marginalised practice's existence. This is pertinent in the context of smart cities, where a limited and homogenized image of an average - male middle class tech-savvy-citizen prevails, mirroring the technological industries that dominate the discourse $[11,30]$.

Political theorist Bonnie Honig uses the notion remainder to refer to the people and practices that are excluded by seeking consensus on an issue [16]. In terms of adversarial design, reconfiguring the remainder is a tactic of "including what is

elaborated beyond a proof-of-concept due to potential legal consequences. For those interested in the topic, the work of Vincent Tan [31] might be of use. 
commonly excluded, giving it privilege, and making it the dominant character of the designed thing" [7]. Shanzhai can thus be seen as a way to reconfigure the remainder; by designing massproduced items for customers that are ignored on the formal market, shanzhai questions the way commercial design practices define the bounds of expected and acceptable behaviour. In other words, shanzhai makes the people that are marginalised in (and through) the prevailing technological imaginaries, visible again.

\section{Hybrid aesthetic}

A second quality of shanzhai is its hybrid aesthetic. Shanzhai products build on existing designs, that are extended and improved with additional functions. This combination of functions is explicitly visible in the design of the product.

The smart bicycle lock still has the appearance of a lock, but also resembles a barcode scanner. This aesthetic emerged during the design process, by building on components that were readily available, for example, the module for scanning and decoding $\mathrm{QR}$ codes. By then exploiting the characteristics of these components, the function of the lock became more explicit and easier to understand. This kind of transparency is often lacking in the smart city, whose infrastructure is difficult to notice and hard to understand [35].

Shanzhai builds on existing designs, but its design language is not related to that of subcultures or hacker aesthetics. Shanzhai leverages the aesthetics of mass-market product design, resulting in objects that look both alien and mundane. This is related to the tactic of articulating in adversarial design, which DiSalvo describes as the capabilities of ubiquitous computing-and by extension, smart cities-to link people, objects and actions together in agonistic collectives [7]. Articulating-or joining-the materiality of different objects together gives new meaning to the constituent elements of a system, leading to new, yet familiar, associations.

\section{Commercial practice}

A third quality of shanzhai is that it is a commercial practice; a "multi-billion USD industry, [...] deeply embedded in contemporary modes of capitalist production" [22]. Yet, shanzhai engages with marginalised groups, through objects whose aesthetics are both strange and familiar; characteristics that are usually assigned to critical or socially engaged strands of design.

As the smart bicycle lock was a prototype, it lacks this third quality of shanzhai. However, by presenting the prototype on an event for start-ups and makers, its perception shifted from a speculative or critical design artefact to an actual product prototype.

In the closing chapter of [7], DiSalvo looks forward to the role of participation in adversarial design. Participation-or the lack thereof-is a pertinent topic in discussions on the smart city [4]. Participation is usually characterised by relatively small-scale projects that aim to profoundly engage stakeholders. Contrarily, shanzhai subverts the possibilities of existing industrial production chains to release small batches of a product, and continuously adapt the design to customer feedback and market response [22]. While this quality of shanzhai should not be read as a call to scale all participation to the level of a global market economy, it is nonetheless an interesting approach that is somewhat unexplored in literature on participation [8].

\section{FURTHER WORK}

This paper has discussed the phenomenon of shanzhai from a design perspective, to explore and articulate the delicate balance between citizen-centric approaches to the smart city that are critical enough to resist assimilation, yet constructive enough to have a sustainable impact. Discussing shanzhai by linking it with the theory on adversarial design allowed us to gain a better understanding of the political qualities of design that could be relevant in the context of smart cities. We believe shanzhai could be a valuable inspiration for designers that work towards citizencentric smart cities and holds promises of providing agency to people or activities that are being left out of consideration in the dominant discourse on smart cities.

The discussion also calls attention to two questions that could give further direction to the aspirations of this paper.

First, while staying and working in Shenzhen for a period of two weeks, the bicycle sharing system caught attention as it is familiar, yet different-both in scale and infrastructure-from the system known to us in European cities. This form of estrangement made it possible to draw on this situation as the context for a prototype. From a design perspective, it is interesting to explore how this outsider's perspective could be recreated inside familiar contexts, in order to identify and engage with local issues. Useful notions for this endeavour could include: work on multispecies ethnography [e.g. 20]; perspectives on care from Science and Technology Studies, now re-emerging in the field of Participatory Design [e.g. 2,11,28]; methodologies on walking [e.g. 29]; or design strategies like archiving and collecting that remind of archaeology [e.g. 14,23].

The second question relates to bringing our work back into a more familiar context, but from a perspective of scale. Shanzhai is deeply embedded in the global market economy and represents a multi-billion industry [22]. It would be worthwhile to identify similar infrastructures on a more local scale and embed projects in those. The prototype of the smart lock, for example, engages with the local issue of sharing bike appropriation by situating it in the start-up community around the Seeed maker space. However, given more time for the project, there could have been a more participatory approach by, for example, engaging with the small temporary kiosks that sell food and commodities near large construction sites; areas were many appropriated bikes could be found.

We aim to explore these questions in future work, by identifying and engaging with local practices that show agonistic qualities. We would like to further elaborate the critical making approach that was used in the design process of the smart lock 
The Shanzhai City

prototype. However, in future case studies, we aim to facilitate more participatory engagements and collaboration in the process.

\section{ACKNOWLEDGMENTS}

This project was partly supported by the Grant Programme for Talent Development of the Creative Industries Fund NL. Thanks to the tutors and students in the Made in China programme of The School of Machines, Making and Make-Believe for the valuable discussions during our time in China.

\section{REFERENCES}

[1] Bastiaan Baccarne, Peter Mechant, Dimitri Schuurman, Pieter Colpaert, and Lieven De Marez. 2014. Urban socio-technical innovations with and by citizens. Interdiscip. Stud. 7. 3, 4 (2014), 143-156.

[2] Maria Puig de la Bellacasa. 2011. Matters of care in technoscience: Assembling neglected things. Soc. Stud. Sci. 41, 1 (February 2011), 85-106. DOI:https://doi.org/10.1177/0306312710380301

[3] Jan Boelen, Ils Huygens, and Heini Lehtinen (Eds.). 2018. Studio time: future thinking in art and design. Black Dog Press, London.

[4] Paolo Cardullo and Rob Kitchin. 2019. Being a 'citizen' in the smart city: up and down the scaffold of smart citizen participation in Dublin, Ireland. Geofournal 84, 1 (February 2019), 1-13. DOI:https://doi.org/10.1007/s10708018-9845-8

[5] Chen-Fu Chien and Jyun-Cheng Wang. 2010. Shanzhai! MediaTek and the "White Box" Handset Market. Harvard.

[6] Regine Debatty. 2017. Shanzhai Archeology: defying our standardized technological imagination. We Make Money Not Art. Retrieved April 8, 2019 from http://we-make-money-not-art.com/shanzhai-archeology-defying-ourstandardized-technological-imagination/

[7] Carl Disalvo. 2012. Adversarial design. MIT Press, Cambridge

[8] Marcus Foth, Martin Tomitsch, Christine Satchell, and M. Hank Haeusler. 2015. From Users to Citizens: Some Thoughts on Designing for Polity and Civics. In Proceedings of the Annual Meeting of the Australian Special Interest Group for Computer Human Interaction on - OzCHI '15, 623-633. DOI:https://doi.org/10.1145/2838739.2838769

[9] Jennifer Gabrys. 2014. Programming Environments: Environmentality and Citizen Sensing in the Smart City. Environ. Plan. Soc. Space 32, 1 (February 2014), 30-48. DOI:https://doi.org/10.1068/d16812

[10] Adam Greenfield. 2017. Radical technologies: the design of everyday life. Verso, London; New York.

[11] Sara Heitlinger (Ed.). 2017. Connected Seeds. Queen Mary University of London.

[12] Garnet Hertz. 2016. What is Critical Making? Current 07. Retrieved February 26, 2019 from http://current.ecuad.ca/what-is-critical-making

[13] Dan Hill. 2013. On the smart city. But what was the question? Retrieved May 26, 2019 from https://medium.com/butwhatwasthequestion/on-the-smartcity-or-a-manifesto-for-smart-citizens-instead-7e0c6425f 909

[14] Natasha Hoare, Giuseppe Licari, and Simone Farresin. 2019. Ore Streams. In Thursday Night Live! Rotterdam. Retrieved September 3, 2019 from https://research-

development.hetnieuweinstituut.nl/sites/default/files/report_design_dialogue s_ore_streams.pdf

[15] Robert G. Hollands. 2008. Will the real smart city please stand up?: Intelligent, progressive or entrepreneurial? City 12, 3 (December 2008), 303-320. DOI:https://doi.org/10.1080/13604810802479126

[16] Bonnie Honig. 1993. Political theory and the displacement of politics. Cornell University Press, Ithaca.

[17] Bailey Hu. Shared Bikes Now More Popular Than Buses in Shenzhen. That's Online. Retrieved August 29, 2019 from https://www.thatsmags.com/china/post/20672/shared-bikes-now-morepopular-than-buses-in-shenzhen

[18] Sebastian Ibold and Christoph Nedopil. 2018. The Evolution of Free-Floating Bike-Sharing in China - Sustainable Transport in China. Sustainable Transport in China. Retrieved August 29, 2019 from http://www.sustainabletransport.org/archives/6278

[19] Michael Keane and Elaine Jing Zhao. 2012. Renegades on the Frontier of Innovation: The Shanzhai Grassroots Communities of Shenzhen in China's Creative Economy. Eurasian Geogr. Econ. 53, 2 (March 2012), 216-230. DOI:https://doi.org/10.2747/1539-7216.53.2.216

[20] S. Eben Kirksey and Stefan Helmreich. 2010. THE EMERGENCE OF MULTISPECIES ETHNOGRAPHY. Cult. Anthropol. 25, 4 (November 2010), 545-576. DOI:https://doi.org/10.1111/j.1548-1360.2010.01069.x

[21] Chuan-Kai Lee and Shih-Chang Hung. 2014. Institutional Entrepreneurship in the Informal Economy: China’s Shan-Zhai Mobile Phones: China's Shan-Zhai
OZCHI'19, December 2-5, 2019, Fremantle, WA, Australia

Mobile Phones. Strateg. Entrep. f. 8, 1 (March 2014), 16-36. DOI:https://doi.org/10.1002/sej.1174

[22] Silvia Lindtner, Anna Greenspan, and David Li. 2015. Designed in Shenzhen: Shanzhai Manufacturing and Maker Entrepreneurs. Aarhus Ser. Hum. Centered Comput. 1, 1 (October 2015), 12. DOI:https://doi.org/10.7146/aahcc.v1i1.21265

[23] Nicolas Maigret, Maria Roszkowska, and Clément Renaud. 2017. Shanzhai Archeology. Inmaterial 2, 117-134.

[24] Peter Mechant, Isabelle Stevens, Tom Evens, and Pieter Verdegem. 2012. Edeliberation 2.0 for smart cities: a critical assessment of two "idea generation"

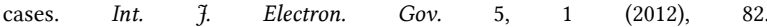
DOI:https://doi.org/10.1504/IJEG.2012.047441

[25] Albert Meijer and Suzanne Potjer. 2018. Citizen-generated open data: An explorative analysis of 25 cases. Gov. Inf. Q. 35, 4 (October 2018), 613-621. DOI:https://doi.org/10.1016/j.giq.2018.10.004

[26] Ivica Mitrović and Oleg Šuran (Eds.). 2016. Speculative - Post-Design Practice or New Utopia? Ministry of Culture of the Republic of Croatia \& Croatian Designers Association.

[27] Chantal Mouffe. 2009. The democratic paradox (Repr ed.). Verso, London.

[28] Matt Ratto. 2011. Critical Making: Conceptual and Material Studies in Technology and Social Life. Inf. Soc. 27, 4 (July 2011), 252-260. DOI:https://doi.org/10.1080/01972243.2011.583819

[29] Stephanie Springgay and Sarah E. Truman. 2018. Walking Methodologies in a More-than-human World: WalkingLab, By Stephanie Springgay, Sarah E. Truman. Routledge, Abingdon.

[30] Yolande Strengers. 2014. Smart energy in everyday life: are you designing for resource man? interactions 21, 4 (July 2014), 24-31. DOI:https://doi.org/10.1145/2621931

[31] Vincent Tan. 2018. Hacking BLE Bicycle Locks for Fun and a Small Profit. In $D E F \quad C O N$ 26. Las Vegas. Retrieved from https://www.youtube.com/watch?v=O-caTVpHWoY

[32] Anthony M. Townsend. 2013. Smart cities: big data, civic hackers, and the quest for a new utopia (First edition ed.). W.W. Norton \& Company, New York.

[33] Matt Ward. 2019. Critical about Critical and Speculative Design SpeculativeEdu. Retrieved September 2, 2019 from http://speculativeedu.eu/critical-about-critical-and-speculative-design/

[34] Jiangning Zhao. 2014. An Argument on the Existing Framework of Entrepreneurship - Shanzhai: An Emerging Entrepreneurial Model. Manag. Adm. Sci. Rev. 3, 5 (July 2014), 762-808.

[35] Liesbett van Zoonen, Fadi Hirzall, Jiska Engelbert, Linda Zuijderwijk, and Luuk Schokker. 2017. "Seeing more than you think": A "Data Walk" in the Smart City. Bang The Table. Retrieved September 5, 2019 from https://www.bangthetable.com/blog/data-walk-in-smart-city/ 